\title{
Building indeterminacy modelling - the 'ZCB Bamboo Pavilion' as a case study on nonstandard construction from natural materials
}

\author{
Kristof Crolla
}

\begin{abstract}
If unprocessed natural materials are the most environmentally friendly construction materials available, how do we develop and communicate design model and construction information that allows dealing with their volatile indeterminacies?

This paper discusses the design and development of the 'ZCB Bamboo Pavilion', a 30-metres-spanning, light-weight, bending-active gridshell from hand-tied bamboo poles, as a case study for the computational design and building information modelling of nonstandard architecture where both applied materials and employed craftsmanship are highly unpredictable in terms of accuracy and precision.

Reflective practice and participatory action research are used to extract knowledge on and challenge the environment of practice, and improve design and construction strategies. The project is used to discuss how traditional construction can be augmented through the strategic injection of computation in the design and construction process, how computation allows for a different mode of collaboration with increased impact for the designer, and how bespoke building information models enable an expanded architectural design solution space.

The paper concludes by arguing for a mode of digital design practice that more proactively operates within a field of real-world indeterminacy. The risk and ambiguity of working with indeterminacies are to be strategically balanced out against idealised digital set-ups and onsite opportunities.
\end{abstract}

Keywords: High-tech versus low-tech, Protocol for error, Bamboo architecture, Bending-active gridshell, Form-finding, ZCB Bamboo Pavilion, Participatory action research, Reflective practice

\section{Background}

Like the relationship between intensive and extensive logics, or the relationship between matter force logics and codification systems, architects are inevitably implicated in the tension between the generative and limiting poles of both. (Reiser \& Umemoto, 2006, p.112)

Demand for responsible eco-friendly architectural practice continues to grow. The search for sustainable, regionally accessible, and renewable materials plays a vital role in reducing the overall carbon footprint of

Correspondence: kristof.crolla@cuhk.edu.hk

Chinese University of Hong Kong, School of Architecture, Room 402, AIT Building, Shatin, New Territories, Hong Kong global building production, especially in the most rapidly urbanising regions of the world. Bamboo is one of the fastest growing natural construction materials and is locally available in most of the developing world, including South America, Africa, and Asia. Certain giant bamboo species suitable for construction grow up to a metre a day and can be harvested in three to 5 year cycles, making bamboo far more sustainable than any wood species.

Although bamboo has been a vernacular construction material for centuries, natural unprocessed bamboo poles are hardly incorporated as a viable structural material in today's construction industry. The plant's unique cellular build-up, however, results in a highly efficient section profile very suitable for use in not only compression or tension, but especially 
when being bent (see Fig. 1). Yet, when used, the material is most commonly employed in processed form or as a traditional wood or steel member replacement. This is partly due to the widely varying dimensional and structural properties of the plant in its natural form, as these for example may differ with varying soil composition or climatic conditions during growth.

This paper uses the design and construction of the 'ZCB Bamboo Pavilion' (see Fig. 2) as a participatory action research (PAR) case study that, through reflective practice (Schön, 1983), aims 1) to improve strategies, 2) modes of practice, and 3) to extract knowledge on the environment in which is being practiced. Concretely, the project illustrates 1) how a traditional, non-digital craft can be augmented through the strategic injection of computation in the design and construction process, 2) how computation allows for a different mode of collaboration in which the role of the designer is expanded to that of construction process engineer, and 3) how alternative usage of building information models can enable expansion of the practice's design solution space.

\section{Participatory action research}

\section{Methodology and aim}

The research component of this project's design and construction is framed in a participatory action research based methodology. The aim is to improve strategies,

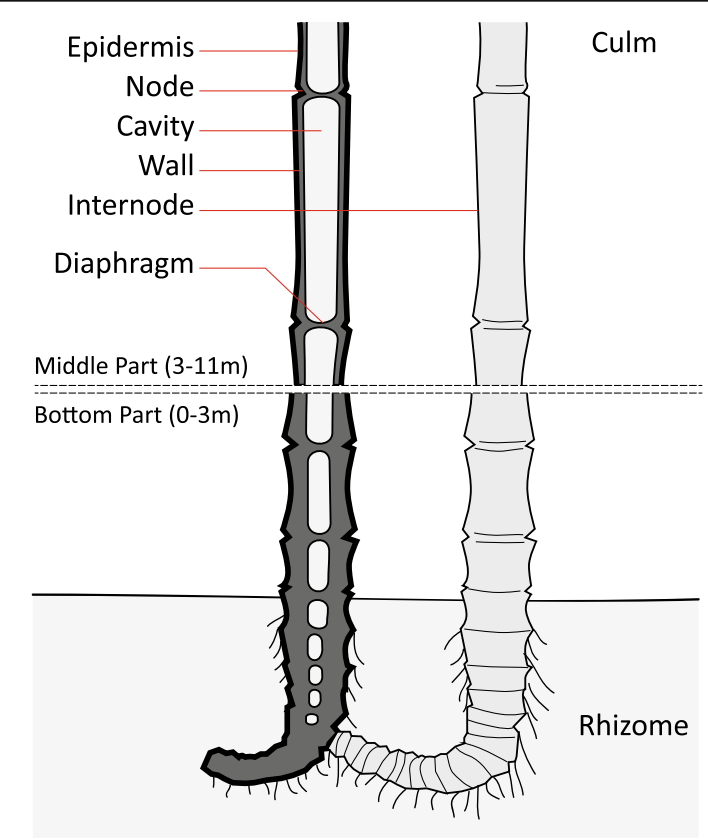

Fig. 1 Bamboo's structural build-up practices, and knowledge of the environment of practice. This method involves combining immediate and specific project problem solution with a reflective process of progressive problem solving. Reflecting on this actively participating in a constantly changing situation is done to enable extracting future alternative guidelines or directions for best practice. Here, this methodology led to proposing new courses of action when dealing with indeterminacy in material or craftsmanship in a digital project design environment.

The project development is set up in a spiral of steps. Each step is composed of a circle of planning, action, and analysis of the results of this action, before advancing the project with more embedded detail. This spiral enabled the crucial components of the final construction to be identified, tested, and included into a flexible design model that encompassed both the digital and physical. Interactive enquiry allowed the balanced problem-solving process to result in a design model that gradually gained concreteness until it was materialised in the final full-scale prototype: the built project.

\section{Local community of practice}

The project team was composed of all parties commonly found in regional practice, including architects, structural engineers, an AP (authorised person), contractors, and specialist consultants. One particular local craft that was incorporated in the team was Cantonese bamboo scaffolding building. In Hong Kong, bamboo has traditionally been used as scaffolding in construction and for the building of temporary event spaces. Without architectural plans or drawings, but by following rules of thumb and century-old hand-tied knots, Cantonese bamboo scaffolders are capable of quickly erecting the large and complex scaffolding structures needed for the construction of the city's many high-rise and other buildings (see Fig. 3).

This locally important and cultural identity defining craft is at risk of being pushed aside by a more easily regulated construction environment using steel scaffolding. Rather than imposing alternative techniques, this research project recaptured the counter-narratives from the bamboo scaffolding tradition and integrated them in an adapted contemporary workflow. Thus, the 'ZCB Bamboo Pavilion' research project investigates how, through the combination of digital design technology, a culturally valuable yet imprecise local craft and an ecologically desirable yet volatile natural material can be practically applied as structural component in lightweiht, sustainable architecture.

\section{Case description}

The 'ZCB Bamboo Pavilion' is a temporary public event space capable of seating 200 people, completed 


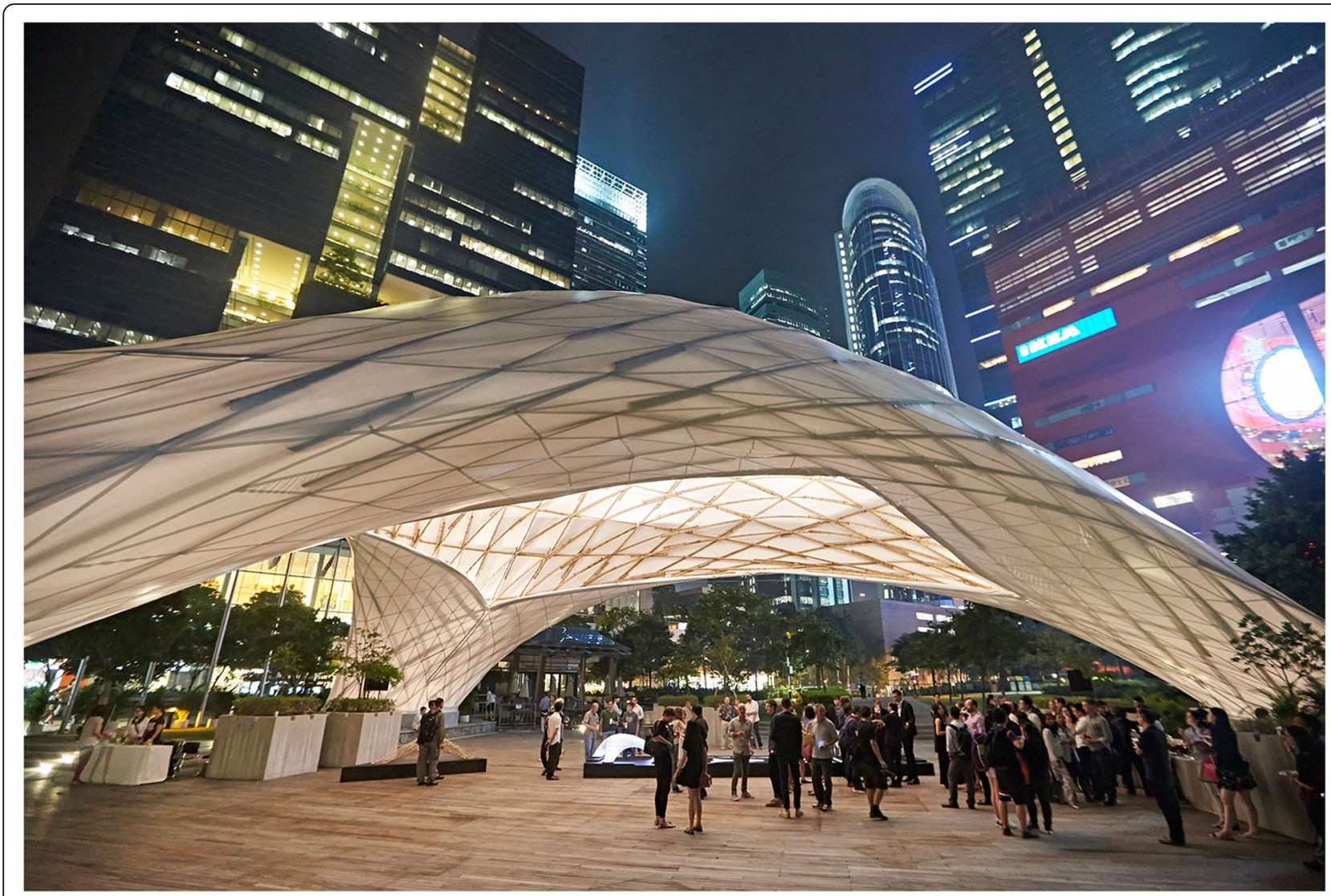

Fig. 2 'ZCB Bamboo Pavilion' during a night-time event

in October 2015 in Kowloon Bay, Hong Kong, where it was used for 8 months before being recycled. It is a bending-active gridshell structure that spans 37 meters, is four stories high, and is wrapped in a lightweight translucent glass-fibre reinforced polymer membrane. Its design development works through immediate and specific problem solution on-the-go until sufficient confidence is built up to initiate final construction.

\section{Design development}

At the start of the research project in early 2014, no building code and hardly any information were available on the use of bamboo as a structural material in architecture, especially not as a bending-active application (Rockwood, 2015). Only reference material on general bamboo architecture was at hand (Jayanetti and Follett, 1988 Janssen and Jules, 2000, Hidalgo-Lopez, 2003, and Velez, 2013).

An iterative series of steps was set up to design and coordinate the project with consultants, client, engineers, and contractors, and to deal with material deviations, construction inaccuracies and human error. Digital procedural design tools, virtual physics simulation engines and physical model prototyping were used at a variety of scales (see Fig. 4). Digital environments were set up to 1) develop a geometry defining workflow from initial physical sketch models, and 2) to procedurally create building information output suitable for deployment in the field. Physical prototyping created opportunities to test and insert newly found information back into the digital model for re-computation and further analysis. This constant dialog between digital simulation and visualisation on the one hand, and analogue testing and physical prototyping on the other, continued throughout the construction and assembly process as serendipitous occurrences required constant design system changes. Except for its scale, the final construction takes no different position from earlier prototypes in this workflow.

The project's structural concept was established through a series of five physical design study model iterations at 1 to 20 scale, made from long bamboo splits (see Fig. 5). Representative geometric behaviour was anticipated by choosing the same material as the final project. Responding to observations on structural stability, connection, and geometry layout, these study models gradually evolved into the final triangulated diagrid shell. This shell is built up from three layers of bamboo and 


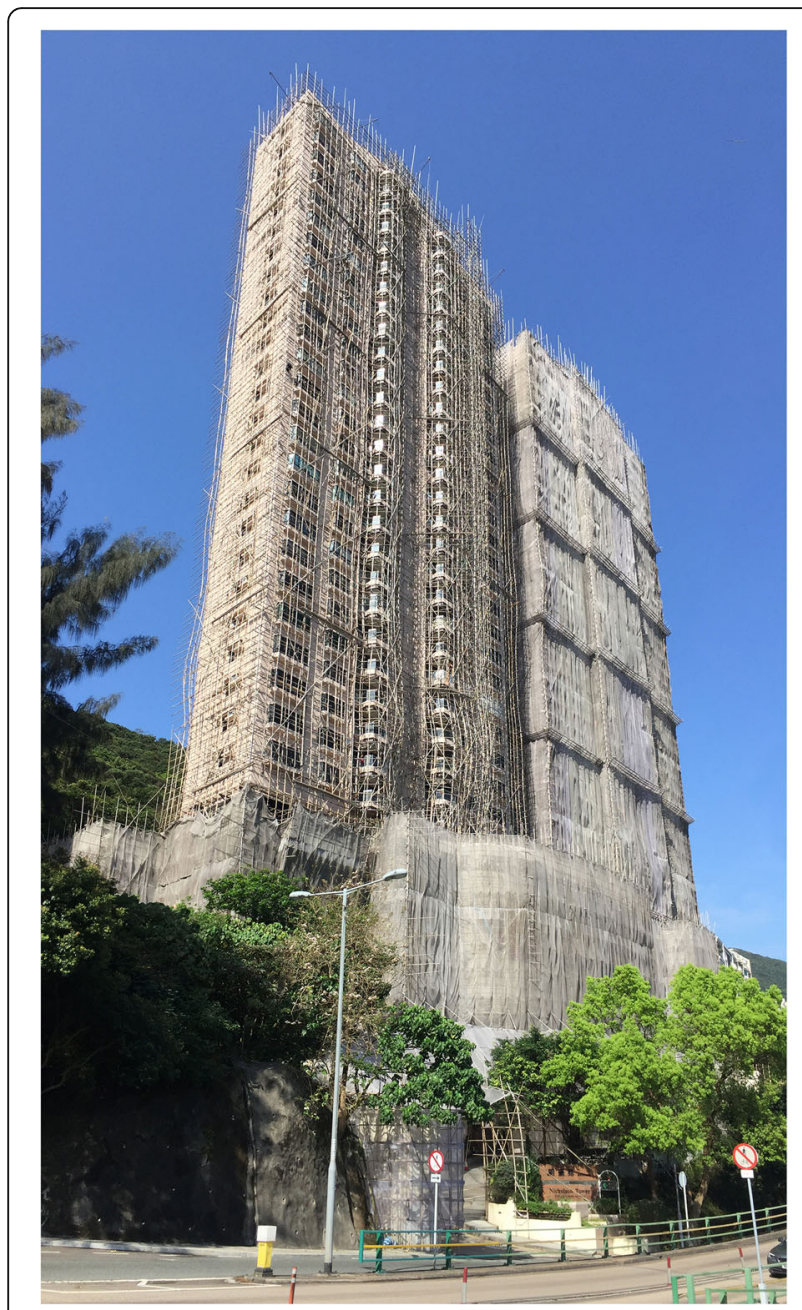

Fig. 3 Hand-tied bamboo used as typical temporary scaffolding in Hong Kong

folds onto itself in three large hollow columns with quadrangulated and free hanging linear members. During model assembly, the bamboo splits were manually elastically bent into shape and connected at the intersection points, defining a fully doubly curved axis model for the overall geometry. Internal bending forces were transferred continuously between members through the knots until a global force equilibrium was

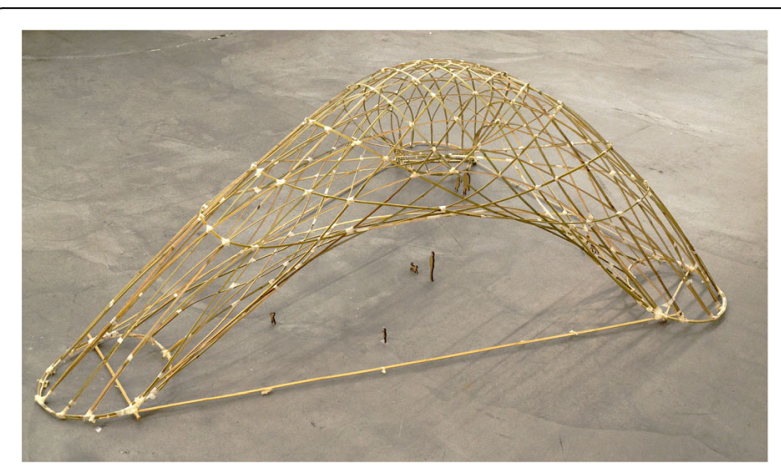

Fig. 5 Original geometry concept study model (bamboo, 1:20)

reached. Thus, a strong and stable lightweight system was created, capable of spanning large distances.

With the fifth physical study model iteration as a basis, a first digital member axis model was developed in McNeel's Rinoceros $^{\circ}$ software environment using procedural modeller plug-in Grasshopper ${ }^{\circ}$ and physical force simulation engine add-on Kangaroo ${ }^{\circ}$ (see Fig. 6). In this model, the bending forces that operate in the physical models were abstracted into corresponding vector forces applied on a discretised curve network represented by a spring-particle system. Each member in this interconnected setup aimed at maintaining its length and straightening itself out. Although all force vectors were defined by abstract numbers with no precise correlation to actual physical material properties, a macro-level behaviour could be perceived that was similar to that found in the physical models. The digital setup would find its force equilibrium in comparable emerging geometries.

The geometry from this first digital model was used to extract conventional architectural plan, section, and elevation drawings for client and contractor communication during public tendering and for operational license applications at a later stage. The model was also used by structural engineers to assess the viability of the project. It further served as a basis for a physical prototype at scale 1 to 30 , made from two metres long bamboo sticks following the geometry axis lines. This prototype used a straightforward mark-up system in which node intersections,

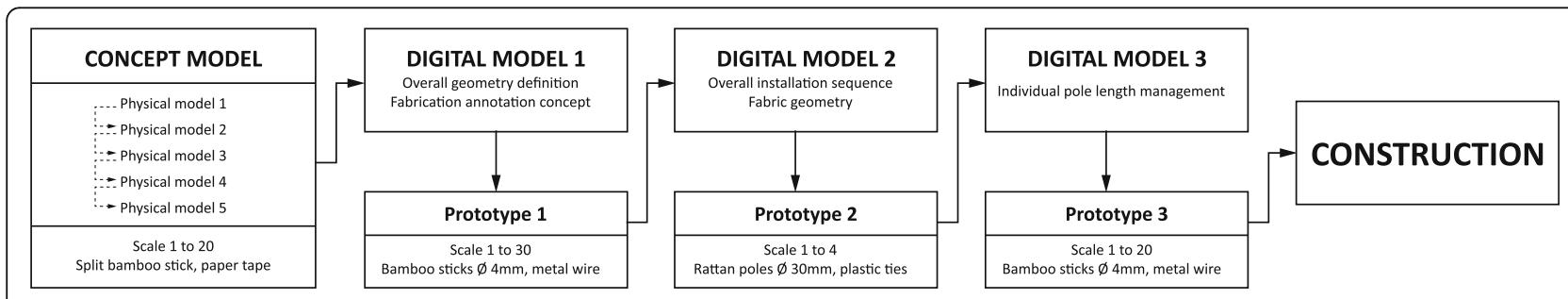

Fig. 4 Design sequence using digital and physical models 


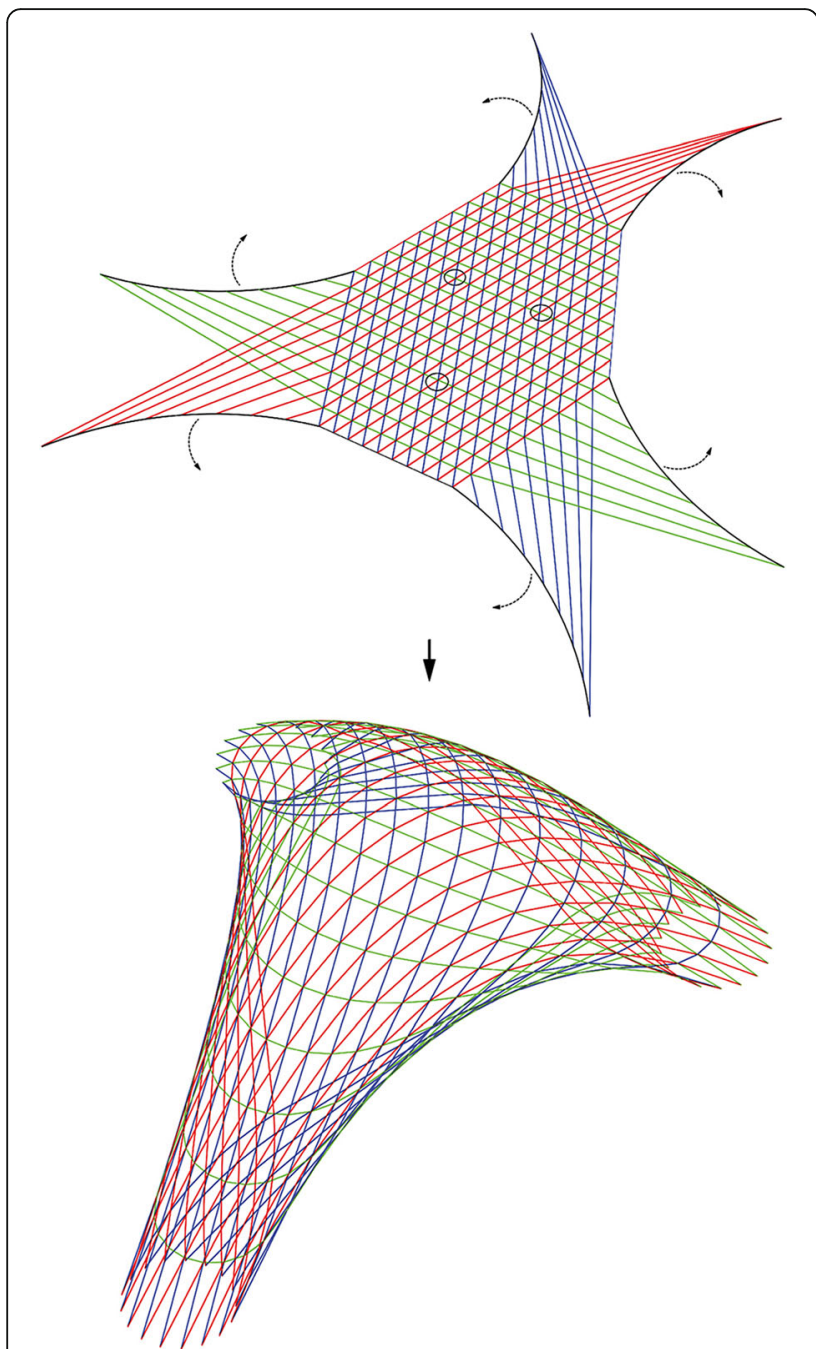

Fig. 6 Digital model 1: spring-particle physics simulation engines are used as equilibrium geometry form-finding tool

extracted from the digital files, were accurately marked up onto long, straight, unbent, bamboo members. Corresponding intersection labels on different members were then brought together one by one while bending the members during the assembly. As matching labels were joined, the form gradually emerged and stiffened up.

The 1 to 30 model revealed several unforeseen challenges that could problematically affect the final fullscale structure. First, in terms of installation sequence, members practically could only be laid up from the top, rather than placed from below (impossible to reach) or woven in (impossible to bend). Secondly, approximation of the layer thicknesses and member shape needed to be more accurately digitally incorporated. Lastly, the two metres length of the used bamboo sticks could not be scaled up thirtyfold onsite. Only roughly the central 12 metres of a bamboo pole is suitable for construction, meaning members would need to be assembled from separate bamboo culms.

A second digital model addressed and incorporated most of these issues. The originally idealised single surface geometry was split up and offset into three layers spaced according to anticipated average bamboo material thicknesses. All members' axis geometry was adjusted to meandered in between only these three layers in a way that followed a carefully orchestrated installation sequence laying-up members from the outside onto previously placed members. This prevented tight bending radii while remaining as close to the original shape as possible.

From this second digital model, a 1 to 4 scale prototype was built. This model was used to assess practicality of improvements in terms of installation sequence and to test large-scale structural behaviour (see Fig. 7). Instead of bamboo, three metres long rattan sticks with diameters of three centimetres were used. Rattan is a reed far more flexible than bamboo, thus exaggerating the prototype's structural behaviour. The long members of the structure were made ahead of assembly by arbitrarily tying rattan sticks together with sufficient overlap to transfer bending forces. Having a correctly scaled diameter, the prototype confirmed the usability of the labelling system and the newly developed installation sequence. The high flexibility of rattan allowed us to assess creep and deflection over time. The outer shell was found to be extremely strong and stable, but the underside of the shell, the 'belly', slumped. In response, a suspension system was introduced into following models. Six points of the 'belly edge line' were suspended from the more stable shell above using metal wire or cable. Furthermore, the arbitrary interconnection of rattan rods into long continuous axis lines revealed knotting challenges in node areas where all three axis

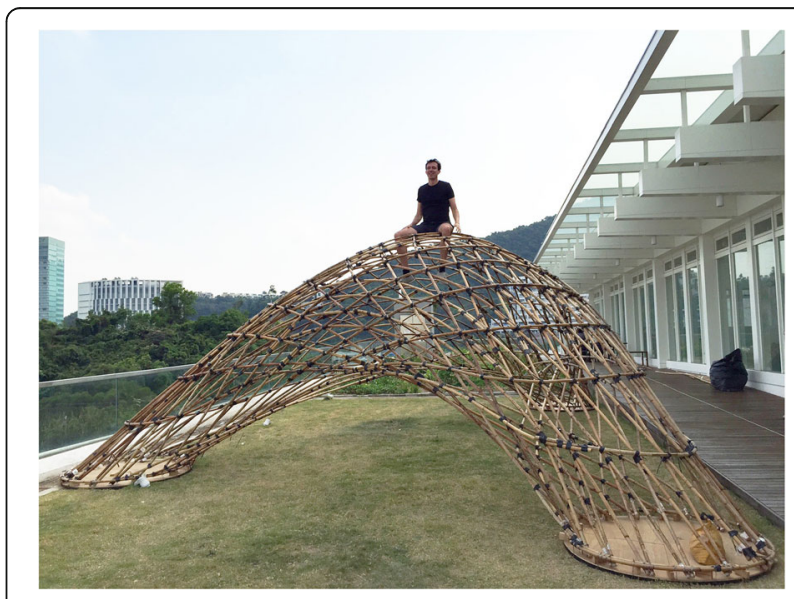

Fig. 7 Second prototype, used for structural behaviour assessment (rattan, 1:4) 
directions happened to have overlaps. Knots had appeared with up to six poles meeting. Also, overlaps close to the end points caused structural difficulties. Hence, the exact positioning of overlaps needed to be included in the following design models.

The final digital model, tested in a third prototype at scale 1 to 20, incorporated all information used for final construction (see Fig. 8). Following a physical maximum bending radius test with several bamboo poles, a digital member curvature analysis was done to check geometry appropriateness. Road transportation restrictions confirmed that the maximum length of bamboo culms that could be transported was $7.2 \mathrm{~m}$, and testing with the actual bamboo poles revealed a needed overlap of $1.5 \mathrm{~m}$ to guarantee proper transfer of bending force without kinking or buckling. This information was incorporated in the model and the pole overlapping pattern was designed. An approximate pole diameter of 12 centimetres was used in the digital models as exact pole diameters ranged from 12 to 15 centimetres at the base and eight to 12 centimetres at the top.

A geometry comparison of a 3D scan of the third prototype and the digital file was made to graphically visualise the similarity between both. This revealed sufficient approximation of the digital emulation model by the physical translation (see Fig. 9). Scaled up, most areas fell within five centimetres of the digital model. Only one area where a model stick broke had a much higher deviation (see blue area in Fig. 10). This provided sufficient confidence in our digital emulation model and implementation methodology to continue with the final step of full scale construction.

\section{Construction information modelling}

Setting up a pliable digital model allowed gradual updating of assumed variables relating to physical material

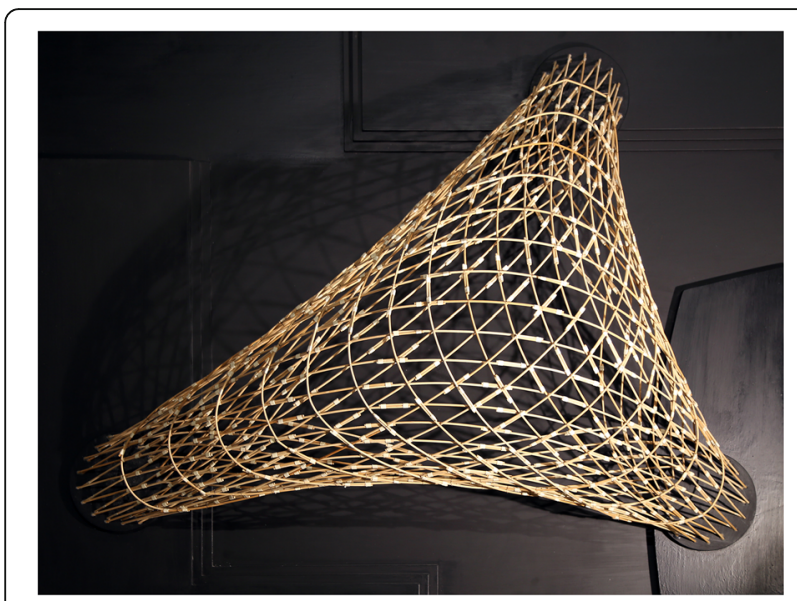

Fig. 8 Third prototype: final installation sequence testing (bamboo, 1:20)

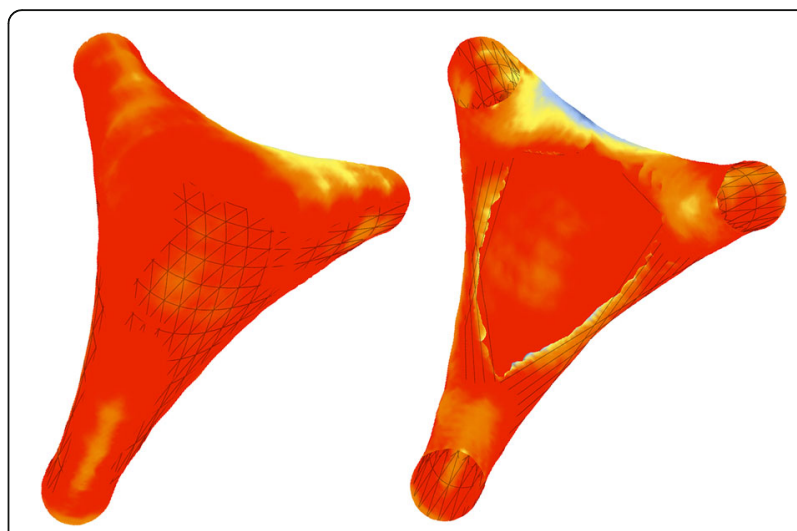

Fig. 9 Comparison between final digital model and scaled-up 3D-scanned third physical prototype: red $=$ less than $50 \mathrm{~mm}$ deviation, blue $=$ more than $1000 \mathrm{~mm}$ deviation; Top view (left); bottom view (right)

properties, construction techniques, detail design, and structural concerns. As data had become more specific throughout the design and prototyping process, the unpredictable nature of both material and assembly method now required for novel construction instructions to be deployed. These needed to be clear enough to minimize any misinterpretation of data, assembly technique, or order of operations, while remaining an open system that could adapt to urgent changes in detail or geometry.

Although the final digital model embedded all the information necessary to automatically produce common graphic representations such as architectural drawings and renderings, only highly selective building information was taken for construction documentation. This reduced the project's complexity into the simplest of
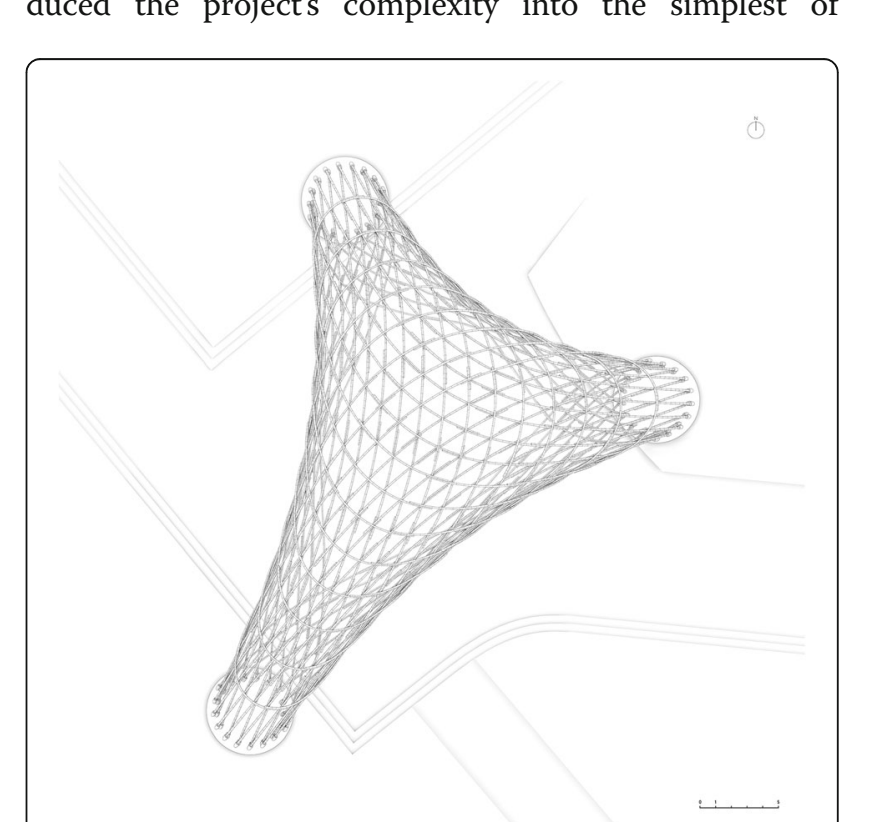

Fig. 10 Digital geometry model 
possible instruction sets. The continuous bamboo axis lines were digitally unrolled into long straight lines. Intersection points with other members were then mapped out onto these lines (see Figs. 10, 11 and 12).

Onsite, the hand-measured marking of these intersection points onto the culms was done using customised sticker labels containing the number information of the poles intersecting at that specific point. The stickers were manually placed on interconnected individual bamboo poles that made up the long continuous members (see Figs. 13 and 14). These members were then lifted on site and manually bent into place. By matching corresponding labels, the overall shape gradually emerged (see Fig. 15).

The construction documents maintained a high level of accuracy in setting out the connection intersection point markings onto members of variable thickness. These markings became guides for an assembly process that included tolerances and human error, resulting in an interconnection that allowed a level of in-situ form finding. The method was straightforward enough to allow for the onsite crossing of language and cultural barriers. A certain level of self-correction took place during the installation: as the system relied on the material's natural bending behaviour, installation errors that deviated too far from the 'exact' simulated digital geometry would become impossible as the real-world material would fight back to find its balance. Large human annotation errors would be spotted and corrected instantly by the spontaneously balanced construction system.

\section{As-built vs. digital model}

The 'ZCB Bamboo Pavilion' was built in an overall seamless construction time of under 90 days. The bamboo structure was realised without any substantial

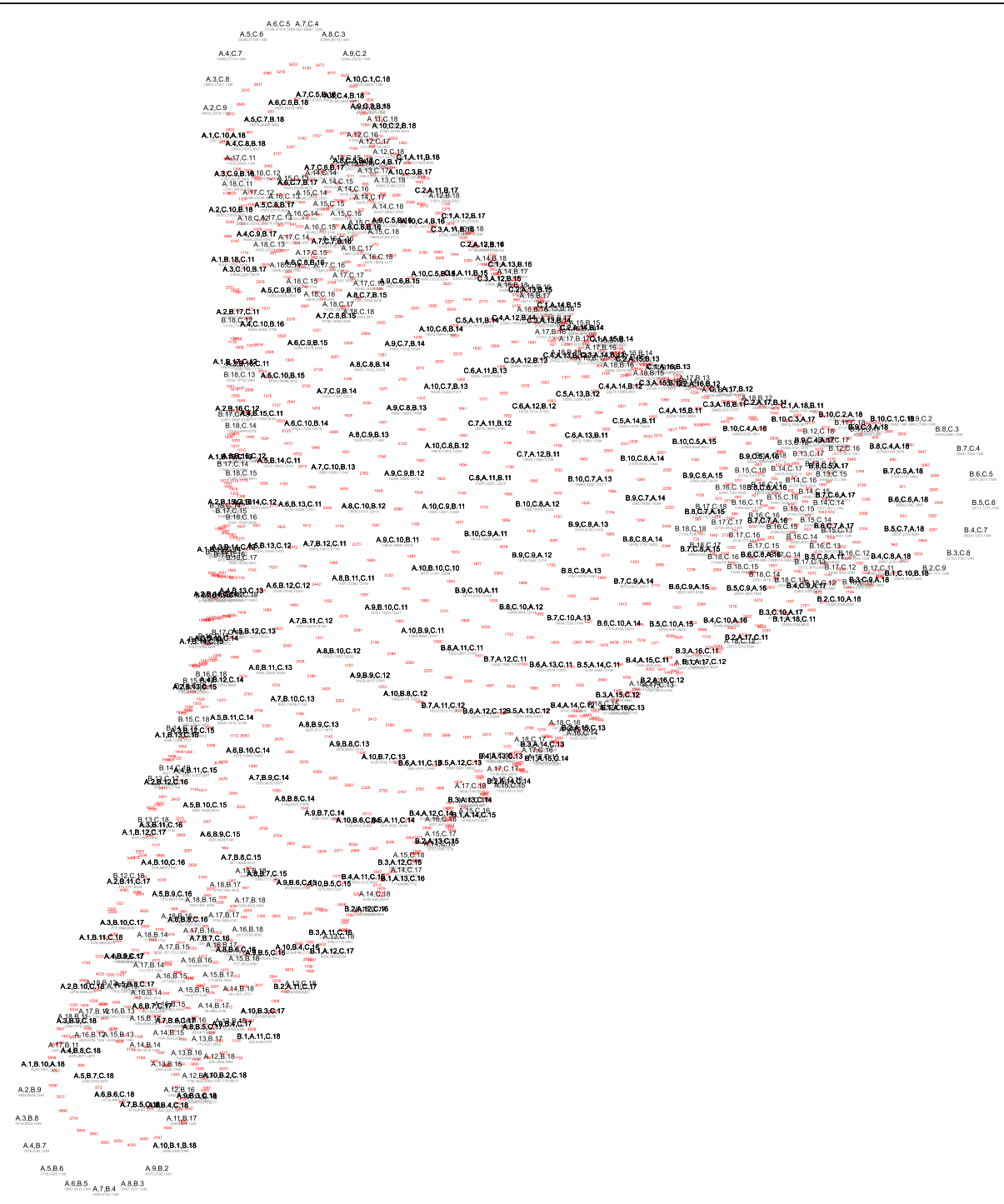

Fig. 11 Pole intersection point coordinates 


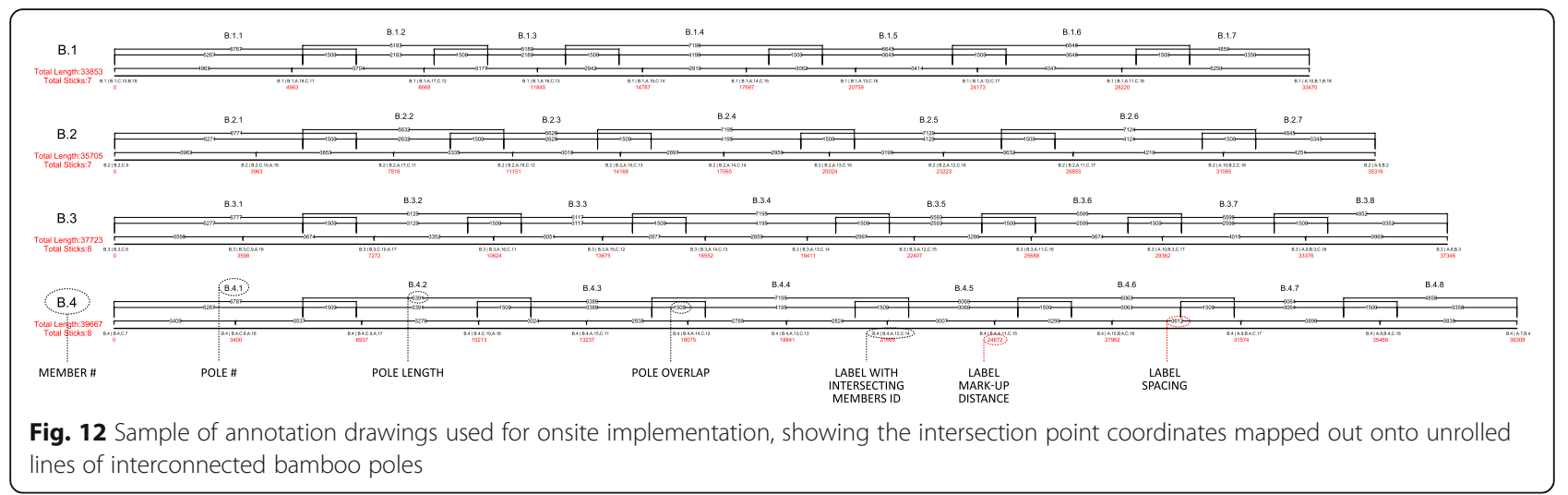

problems using the same construction methodology as used in the small-scale prototypes, and using the same fixing techniques and materials found in the traditional local craft. Only in areas of tightest bending radii were additional notches in the culms needed to meet the required curvature - an unusually hot summer had dried and stiffened the onsite poles to an unanticipated degree. The construction documentation system worked well to direct contractors with a clear set of instructions and order of operations for assembly. Stable grid-shell geometry gradually emerged as each member was bent and fixed onsite (see Fig. 16). Natural dimensional variations of the bamboo and tolerances in the knots, however, made it impossible to perfectly match each label everywhere as cumulative slippage occasionally built up (see Fig. 17). Also, members sporadically were too short or too long at their foundation connection points due to added deviations, requiring ad hoc detailing solutions. The hyper-precise pole intersection coordinate points from the digital emulation model (see Fig. 12) eventually materialised into an 'approximative sphere' measuring roughly $20 \mathrm{~cm}$ in diameter.

Like the geometry comparison between the 1 to 20 scale physical model and the digital model, a high-resolution

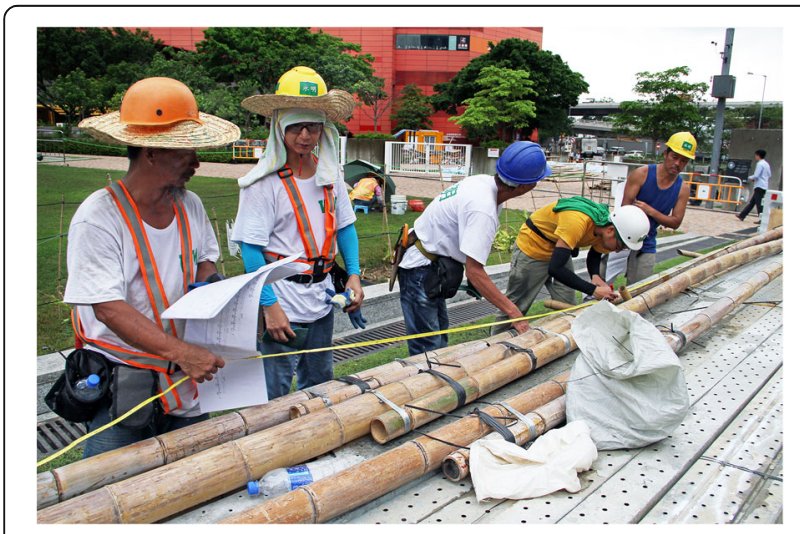

Fig. 13 Bamboo poles are interconnected into long continuous members
3D scan was made of the final built geometry and measured against the digital data (see Fig. 18). Most bending members found their final form within 50 millimetres from the simulated model. In two areas of minimal curvature, however, the structure attempted to flatten out with two of the three members straightening up more than anticipated, resulting in deviations of over 1 metre. This spontaneously occurred deviation was deemed acceptable as the synclastic nature of the surface in those areas of the surface remained. This meant that no local buckling or amplified continuous creep under the anticipated load was to be expected. Also visually, the difference did not pose any problems as the public still perceived the form to be in its pristine platonic shape. Thus, the deviation was considered part of the project's natural form finding behaviour. Considering the complexity of the project, the limited amount of time on site, its unique digital design and documentation process, and the experimental nature of assembly, the overall construction was concluded to be successful.

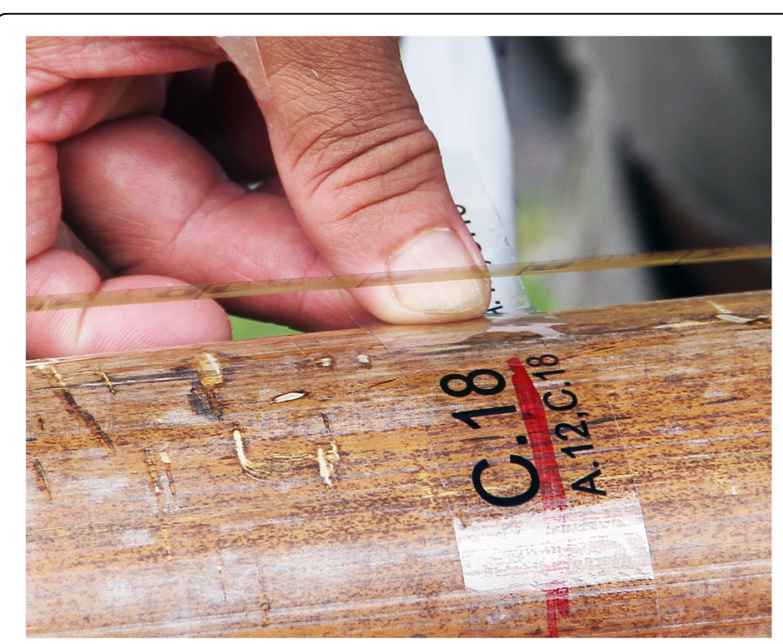

Fig. 14 Sticker labels with connection information are applied using a measuring tape 


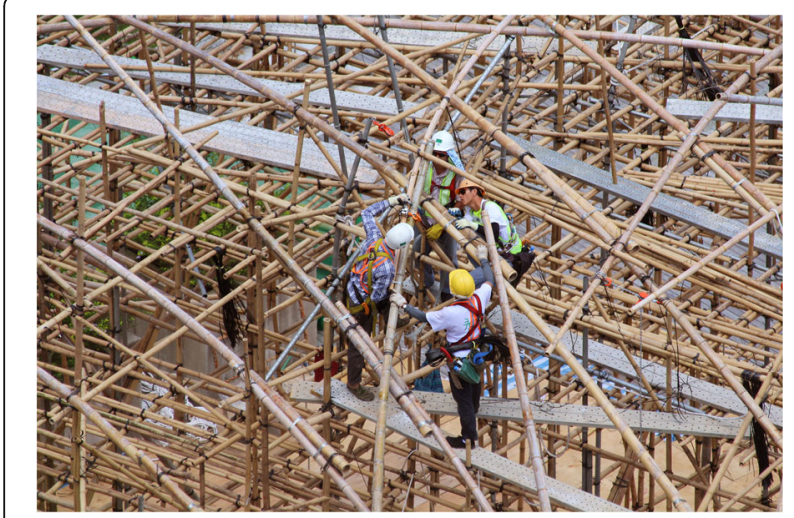

Fig. 15 Onsite installation by matching corresponding labels

\section{Building indeterminacy modelling}

Since a gap between the BIM and the building is inevitable and potentially advantageous, the question remaining is: how big a gap should there be? (Willis \& Woodward, 2010, p. 184)

The 'ZCB Bamboo Pavilion' challenges the role building information models (BIM) can play in facilitating alternative architecture production. Although traditional architectural drawings and visualisations were extracted from earlier models for communication and licensing purposes, for construction itself the focus lay on managing selective unconventional types of data. BIM's purpose is hereby pushed to go beyond the optimising and streamlining of generic building production. Since there will always be a gap between digital and real-world environments (Willis and Woodward, 2010), tolerances always will continue requiring being absorbed in the design somewhere. But what if these tolerances are an order of magnitude larger than what is common practice? Or what happens if the structure itself is expected to drastically move, and change during the construction process as it finds its equilibrium form?

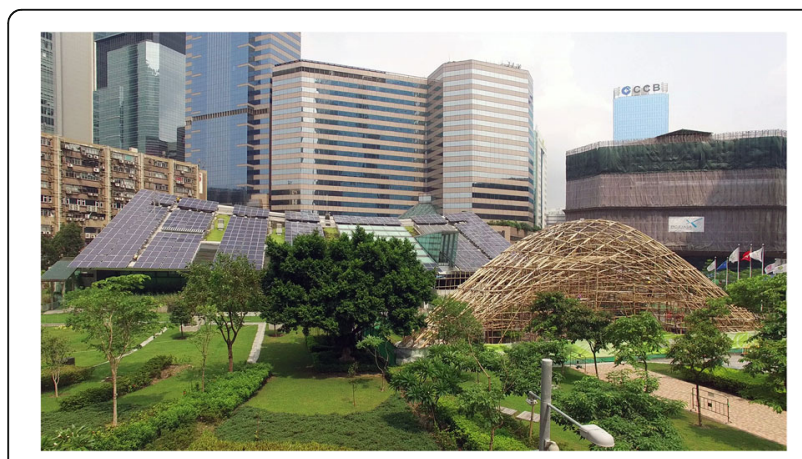

Fig. 16 Completed bamboo structure

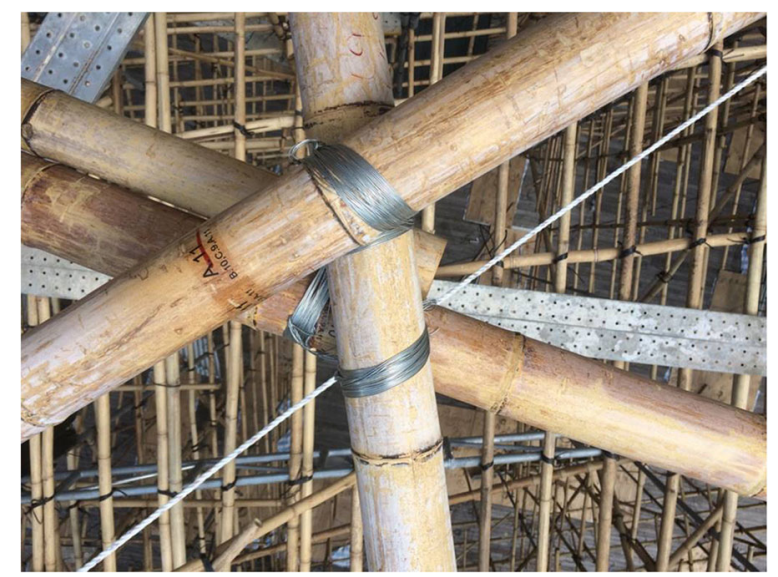

Fig. 17 Typical example of labels mismatch

A fully accurately detailed and precise BIM model that aligns with conventional building models could hypothetically be created for the 'ZCB Bamboo Pavilion', offering full control and predictability over all involved materials, components and their tolerances. To set up this model, each bamboo pole would need to be scanned and measured ahead of time at high accuracy. That bespoke data set would then be brought into the virtual environment. A live recording of the pole behaviour would feed back into the system in real-time as poles are bent and fixed in place onsite, allowing for constant recalculation and assessment, and so forth.

There would be advantages to this hypothetical model. Embedded digital monitoring could display or predict abnormal or undesirable material deflections or deformations and could suggest in real time where adjustments need to be made. Yet, although probably technologically feasible, this level of control is currently not practical nor cost effective. More importantly, ultimately it is not necessary and redundant. The level of

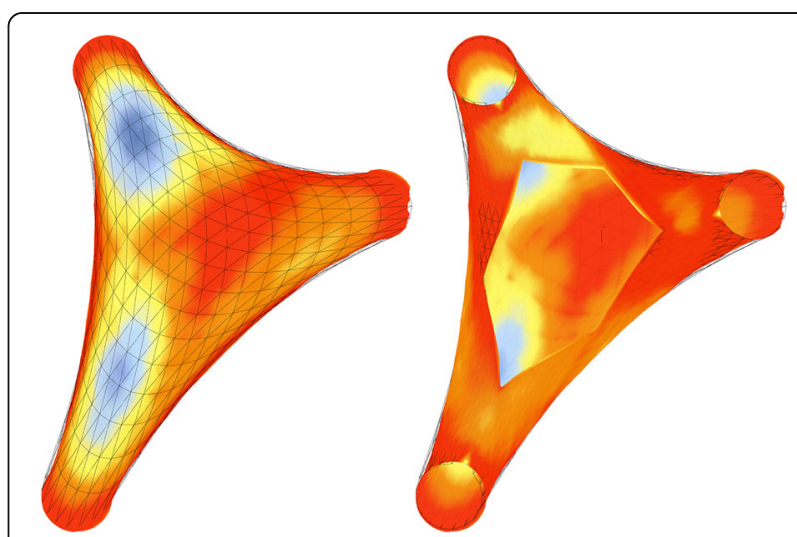

Fig. $183 D$ scanned fabric vs. originally designed fabric: red = less than $50 \mathrm{~mm}$ deviation, blue $=$ more than $1000 \mathrm{~mm}$ deviation; Top view (left); bottom view (right) 
sophisticated and automated decision-making this model would bring is currently already to a large extend embedded in construction implementation, as the design and construction team can rely on experience, intuition, and common sense to solve unforeseeable issues on-thego. The gap between the virtual and the real can never be fully sealed. The role of physical prototyping and material testing and the inclusion of their findings into a flexible digital model hence needs to be valued as essential.

If unprocessed natural materials are to be incorporated more pervasively in construction, then alternative design information models and implementation systems are needed that allow dealing with their volatile indeterminacies. This project eventually positioned the digital emulation model not as an exact, ideal, and finished model that needs real-world replication, but as an element in a chain of back-and-forth between simulation and prototyping. The digital model should embody variability so that its physical materialisation can lead to singular expression. Unavoidable serendipitous occurrences could thus be exposed and incorporated more effectively. This shifts the BIM model's focus from a quantification and simulation tool to a much more open-ended environment that works with the peculiarities of the material world rather than in isolated abstraction ahead of construction.

\section{Discussion and evaluation}

\section{Permitting natural variability}

The 'ZCB Bamboo Pavilion' pioneers in pushing the boundary of which 3D model based data transactions are incorporated in construction. As highlighted, certain benefits can only be found in more detailed or alternative BIM models. However, the information modelling tools most commonly used in practice are not developed to accommodate the variety of material inaccuracies, tolerances, and indeterminacies faced onsite. Although digital models are commonly updated to achieve a final "as built" model, this information flow does not incorporate the indeterminacy back into the design process. This can result in a final model which potentially dramatically differs from original design intent. The assimilation of naturally variability in digital environments is not possible with conventional tools but would be a requirement if we want to use raw materials that don't undergo extensive industrial standardisation.

\section{Open source API}

The ability for building information modelling technology to move forward and improve in areas such as indeterminacy could be facilitated by the incorporation of a more open-source application program interface (API). Such an interface can allow for greater direct access to database fields and tables, enabling designers to take more direct and spontaneous control of their model data. This open interface then allows direct access to the software for autonomous development of more accurate customised tools that could be configured with greater sensitivity toward material attributes and structural behaviour beyond convention. An open-source attitude, whereby the appropriation and sharing of content is encouraged, will greatly enhance the versatility of BIM managers in areas of design, allowing them to introduce solutions more comprehensively to construction indeterminacy by developing their own ad-hoc solutions. To some extent, this API is becoming available in the form of scripting languages such as Python ${ }^{\circ}$, Visual Basic ${ }^{\circ}$ and graphical versions such as Dynamo $^{\circ}$ or Grasshopper ${ }^{\circ}$, which are, albeit somewhat proprietary in nature, steps in the right direction.

\section{Analogue/digital futures}

The developed workflow discussed in this paper provides a very timely practice-based working model to deal with unforeseen in situ occurrences. Architectural designers now globally have easy access to cheap computational power to allow its practical incorporation even in smallscale projects. Sufficiently powerful software became accessible and versatile enough to allow for bespoke customisation in one-off designs. In the meantime, today, still, the majority of onsite construction is done almost exclusively by hand, onsite, without the use of sophisticated construction and fabrication technology, relying on whatever craftsmanship or skill is locally available. These two realities need to be brought in close dialogue.

The 'ZCB Bamboo Pavilion' has pushed the boundaries of the design solution space of bamboo architecture. It's mode of practice, though, could be employed into the arenas of other analogue construction systems as well. Through setting up a mutually enforcing feedback loop between the digital and physical world and relaxing the rigidity of digital design models, a geometrically, structurally, and spatially wider range of architecture will become possible.

While technological conditions continue to advance rapidly, the material and digital worlds need to be brought closer together through a balanced workflow. In this, the architect's fundamental task is the definition within the overall design and construction process of the comfortable balance between what digital tools allow for and what is appropriate onsite.

\section{Conclusion}

As a participatory action research project, the 'ZCB Bamboo Pavilion' (see Fig. 19) illustrates how strategies, modes of practice, and understandings of the environment of practice can be identified and with computation be adapted and expanded to allow the extension of the 


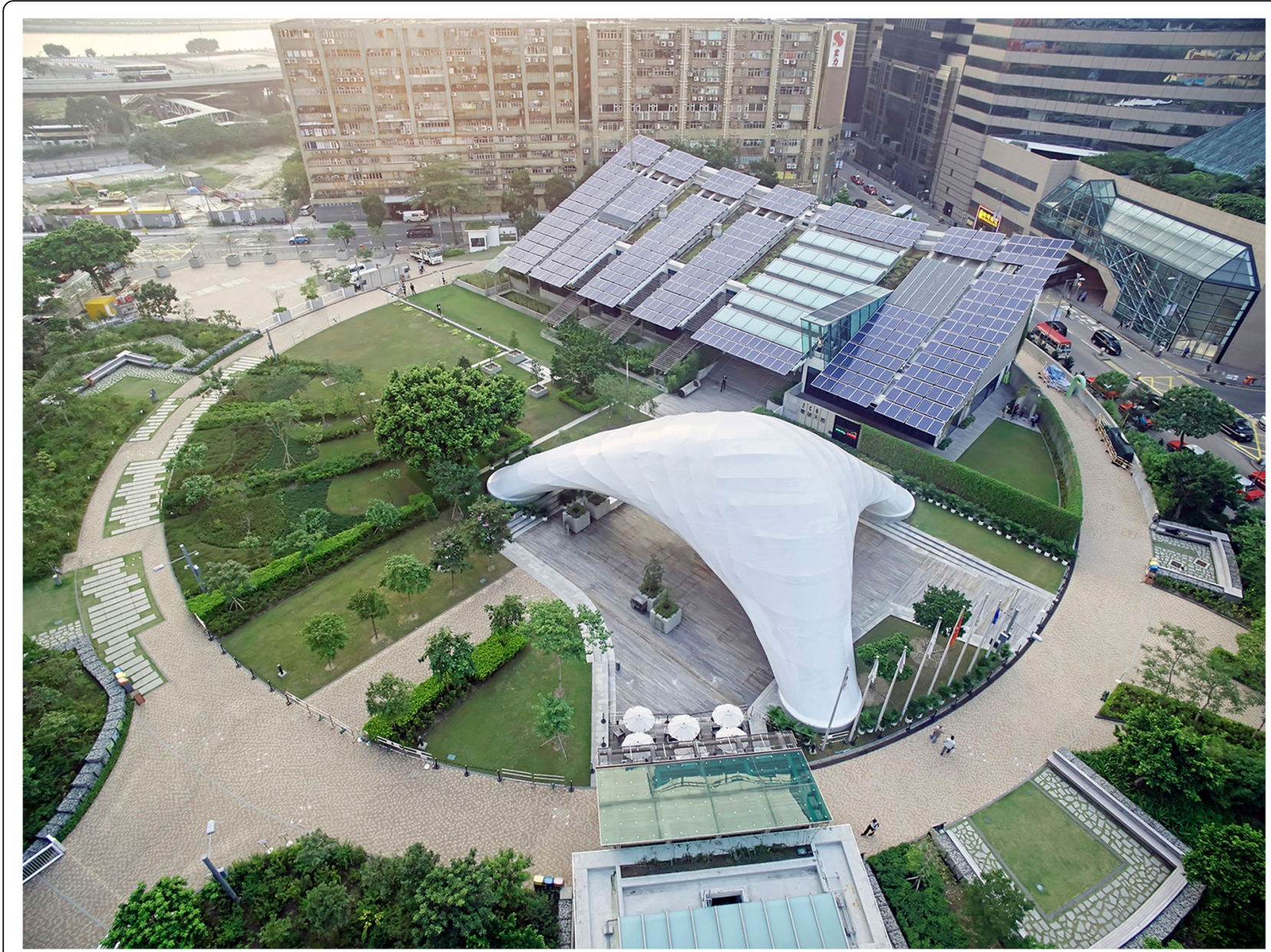

Fig. 19 Aerial view of 'ZCB Bamboo Pavilion' by day

architectural design solution space. The project is an experiment in the ability to manage onsite expectations based on an initial set of unknowns within a construction system. Through time, investigation, refinement, and development using both physical prototypes and digital models, gaps in the design process were clarified and reinserted into the workflow. Information gained on-the-fly thus became part of ongoing simulations eventually capable of anticipating volatile system behaviour.

Paramount to the project's success was a digital configuration capable of flexibility with respect to the tolerances of real-world materials and onsite assembly techniques, as well as the conception of the final project as a self-correcting analogue material computer that takes over when the digital emulation no longer is applicable. For this, alternative types of construction documentation and reference material extracted from the complex original data needed to achieve a level of practical simplicity.

The adoption of digital tools needs to be applied sensitively to accommodate and take advantage of a wider array of raw materials and regional labour skills. The relationship between absolute accuracy and a system with indeterminacy will remain open as the adoption of natural materials and varied construction practices are further explored. Through the innovative use of computational tools in architectural practice, customised building information models can adopt an ad-hoc approach toward balancing risk, probability, and ambiguity against an idealized design.

\section{Abbreviations}

AP: Authorised person; API: Application program interface; BIM: Building information model; PAR: Participatory action research; ZCB: Zero carbon building

\section{Acknowledgments}

Design research team: Principal Investigator, CUHK: Prof. Kristof Crolla; CoInvestigator, CUHK: Mr. Adam Fingrut; Research Assistants, CUHK: Mr. IP Tsz Man Vincent, Mr. Lau Kin Keung Jason; Consultants: Dr. Goman Ho and Dr. Alfred Fong (Structural Engineering), Mr. Vinc Math (Bamboo Consultant); Authorised Person: Mr. Martin Tam; Registered Structural Engineer: Mr. George Chung; Project Documentation: Mr. Ng Ka Hang Kevin, Mr. Grandy Lui, Mr. Michael Law and Mr. Ramon van der Heijden.

CIC/ZCB Client Project Team: Executive Director, CIC: Dr. Christopher To; Publicity, ZCB: Ms. Yan Ip; Technical Services, ZCB: Dr. Margaret Kam. 
Construction Team: Main Contractor: W.M. Construction Ltd.; Bamboo Construction: Sun Hip Scaffolding Eng. Co., Ltd.; Fabric Contractor: Ladden Engineering Ltd.; Lighting: CONA Technology Co. Ltd. \& Brandston Partnership Inc.

\section{Funding}

This Chinese University of Hong Kong (CUHK) School of Architecture project research was partially supported by a grant from the Research Grants Council of the Hong Kong Special Administrative Region China: Project No. CUHK244001 14, Project Title: Architectural design and building of lightweight, bending-active, bamboo shell structures for Hong Kong, using live physics engines.

\section{Author's contributions}

Kristof Crolla is the sole author of this paper.

\section{Author's information}

Kristof Crolla is a Hong Kong-based licensed architect and Assistant Professor in Computational Design at the Chinese University of Hong Kong, School of Architecture. His research focusses on the strategic implementation of computation in architectural design. He graduated Magna Cum Laude as Civil Architectural Engineer from Ghent University and practiced in Belgium before moving to London in 2005 to attend the Architectural Association School of Architecture (AA)'s Master of Architecture programme Design Research Laboratory. Following this, he worked for several years for Zaha Hadid Architects, while teaching in parallel at the AA and other institutions worldwide. Since 2010, he is based in Hong Kong where he set up his practice Laboratory for Explorative Architecture \& Design Ltd. (LEAD). He has been invited as a jury critic, lecturer, and tutor in numerous institutions throughout Europe, Asia, Chile and South Africa. He is best known for projects such as 'Golden Moon (Hong Kong, 2012)' and 'ZCB Bamboo Pavilion (Hong Kong, 2015)', which internationally received over two dozen design awards and accolades, including the G-Mark (Japan), Architizer A+ (USA) Awards, and most recently the 2016 World Architectural Festival Award - Small Project of the Year 2016, nicknamed «The Architectural Oscars».

\section{Competing interests}

The author declares that he has no competing interests.

\section{Publisher's Note}

Springer Nature remains neutral with regard to jurisdictional claims in published maps and institutional affiliations.

Received: 16 January 2017 Accepted: 30 June 2017

Published online: 21 July 2017

\section{References}

Hidalgo-Lopez, O. (2003). Bamboo: The gift of the gods. Bogotá: O. Hidalgo-López. Janssen, \& Jules, J. A. (2000). Designing and building with bamboo. Eindhoven: International Network for Bamboo and Rattan (INBAR).

Jayanetti, D.L. and P.R. Follett. (1988). Bamboo in construction: An introduction. Buckinghamshire: TRADA New Delhi: International network for Bamboo \& Rattan /dept. for international development.

Reiser, J., \& Umemoto, N. (2006). Atlas of novel tectonics. New York: Princeton Architectural Press.

Rockwood, D. (2015). Bamboo Gridshells. London: Routledge.

Schön, D. (1983). The reflective practitioner: How professionals think in action. London: Temple Smith.

Velez, S. (2013). Architecte // la maitrise du bamboo, architect // mastering bamboo. Arles: Actes Sud.

Willis, D., \& Woodward, T. (2010). Diminishing difficulty: Mass customization and the digital production of architecture. In R. Corser (Ed.), Fabricating architecture, selected readings in digital design and manufacturing (pp. 178-213). New York: Princeton Architectural Press.

\section{Submit your manuscript to a SpringerOpen ${ }^{\circ}$ journal and benefit from:}

- Convenient online submission

- Rigorous peer review

- Open access: articles freely available online

- High visibility within the field

- Retaining the copyright to your article 